\title{
How to implement a smoke-free policy ${ }^{\dagger}$
}

\author{
Irene Cormac \& Lisa McNally
}

\begin{abstract}
It takes courage, leadership and planning to successfully implement a smoke-free policy in mental health settings. The content of the policy is crucial in setting parameters for implementation. Management and clinicians should work closely together to develop and coordinate the implementation strategy, ensuring that resources are effectively used and deadlines are met. Key success factors are effective management at both central and local levels, as well as consultation with service users, carers and staff to gain support for the policy and obtain suggestions for improvement. Other important factors are advance planning, recruitment of experienced staff, effective communication and extensive training of staff in smoking cessation support. Local teams should develop appropriate procedures based on the policy. They should work closely with the central management team. The resources developed and obtained by local teams should be shared throughout the organisation and should be tailored to meet the needs of particular services.
\end{abstract}

Smoking has been an accepted part of the culture of mental health units for many years. Studies have shown that $74 \%$ of psychiatric in-patients are smokers (Meltzer et al, 1996), compared with $24 \%$ of the general population (Office for National Statistics, 2007). Mental healthcare staff are more than twice as likely to be smokers as their colleagues in general healthcare (Trinkoff \& Storr, 1998). Mental healthcare staff are also known to be significantly less positive towards smoking-related policies and treatments than their counterparts in other areas of healthcare. A recent UK survey reported that roughly 1 in 10 staff in general healthcare settings disagreed with a smoking-ban in their wards or clinics; however, 1 in 3 psychiatric staff were against such a ban in their settings (McNally et al, 2006).

In the UK and Ireland, smoke-free legislation has been introduced which bans smoking in enclosed public areas or workplaces (including vehicles) as follows: the Health Act 2006 for England and Wales; the Public Health (Tobacco) (Amendment) Act 2004 for Ireland; and the Smoking, Health and Social Care (Scotland) Act 2005. In Scotland and Ireland, mental health units are exempt from implementing smokefree legislation. Guidance for voluntary smoke-free implementation in mental health units is available for Scotland (Griffiths, 2005) and for Ireland (Irish Health Promotion Network, 2006).

${ }^{\dagger}$ See pp. 208-216 and 217-228, this issue.
The Health Act 2006 applies to England and Wales, and grants authority to make regulations respectively to the Secretary of State for England and to the National Assembly of Wales. The Smokefree Premises etc. (Wales) Regulations 2007 provide exemption to mental health units from being smokefree (para. 4, section a). However, in England, the Smoke-free (Exemptions and Vehicles) Regulations 2007 gave only a temporary 1-year exemption to mental health units. This was in light of consultation responses from many who argued that it is unethical to expose detained patients and staff in mental health settings to the harmful effects of passive smoking. The principle of the human right to live or work in a safe environment prevails over the rights of individuals to smoke (Campion et al, 2006).

Accordingly, from 1 July 2008 in England smoking will not be permitted in any NHS buildings or vehicles. This applies to patients, staff, visitors and contractors working on the premises. National Health Service staff will not be permitted to smoke when on NHS trust business, for example visiting patients in the community.

Northern Ireland is covered by the Smoke-free (Exemptions, Vehicles, Penalties and Discounted Amounts) Regulations (Northern Ireland) 2007, which came into operation on 30 April 2007. As in England, these regulations provide a time-limited exemption of 1 year for mental health units, during which smoking may be allowed in a designated smoking room. After 30 April 2008, smoking will no longer be allowed within any enclosed spaces.

Irene Cormac is a consultant forensic psychiatrist with a special interest in physical healthcare at Nottinghamshire Healthcare NHS Trust (Rampton Hospital, Retford DN22 0PD, UK. Email: irene.cormac@nottshc.nhs.uk). She led the clinical implementation team at Rampton Hospital during the introduction of the smoke-free policy. Lisa McNally is a chartered health psychologist employed by Wandsworth Primary Care Trust's Public Health Department in London. She is an expert in smoking cessation and smoke-free policies in mental health settings. 
The implementation of smoke-free policies in mental health settings represents a significant challenge (Jochelson, 2006). Staff fear patients' reactions to 'no-smoking' rules, feel unsure how to help patients who are smokers and believe that there will be adverse consequences such as violence. They are afraid that they will not be able to cope with the challenges.

These fears are understandable but research evidence does not support them. A review of 22 studies of total and partial smoking bans in mental health settings found no adverse effects in terms of unrest or refusal to comply (el-Guebaly et al, 2002). Lawn \& Pols (2005) reviewed 26 international studies of smoking-bans in mental heath units, finding no increase in aggression, use of seclusion, discharge against medical advice or use of medication on an 'as required' basis.

Staff attitudes can become more positive, as shown by Resnick \& Bosworth (1989), who found that initially only $7 \%$ of staff favoured a smoking ban before its introduction, rising to $90 \%$ postimplementation. In a Canadian study, successful smoke-free implementation was found to depend on staff preventing patients from gaining access to tobacco (Harris et al, 2007).

In this article we describe key elements of an effective smoke-free policy and practical ways of implementing such a policy applicable to a wide range of mental health settings.

\section{First steps and strategic decisions}

\section{Consultation}

Before making the transition to a smoke-free environment, consultation should take place widely with service users, carers, staff and union representatives of staff. It is important to seek support from opinion leaders, lead clinicians and management throughout the organisation. Information should be shared on the serious health risks of tobacco smoking, the benefits of a smoke-free environment and the successful implementation of smoke-free policies in various psychiatric settings. As a focus for discussions, it is helpful to have a draft smoke-free policy, which can be adapted to meet the needs of the organisation. Legal advice should be obtained about any potentially contentious issues.

\section{Content of a smoke-free policy}

All policies must comply with legislation pertaining to the jurisdiction of the site of the mental health service concerned. In the first paragraphs of the policy, reasons should be outlined for its introduction (Box 1).
Box 1 Reasons for the introduction of a smoke-free policy

- Smoking causes damage to the health of individuals, those around them and their unborn children

- Smokers do not have the right to damage the health of others by smoking

- Smoking is a fire hazard

- Patients in mental health services have the right to be treated in a smoke-free environment

- Employees have the right to work in a smoke-free workplace

- Healthcare organisations have a duty to provide a safe and healthy environment

- Legislation makes it an offence to smoke in an enclosed public space

\section{Partial or total ban on smoking}

It is crucial to decide whether grounds as well as buildings will be smoke-free. Examples of pros and cons are listed in Box 2. A total ban may be easier to implement in mental health settings, with less adverse effect on ward functioning (Lawn \& Pols, 2005). Partial bans permitting smoking in outside areas can be well accepted (Etter \& Etter, 2007), but patients will need access to such areas. The health risks from passive smoking indoors are well documented, but there is less evidence of the effects of passive smoking on non-smokers outdoors. However, in Ireland a study on bar staff exposed to smoking in outside areas showed that although the risks of passive smoking were significantly reduced, they were not fully eliminated (Mulcahy et al, 2005).

\section{Smoking breaks}

In a partial ban, staff can supervise planned smoking breaks for patients more easily than 'on-demand' smoking. Patients can participate in negotiations regarding the timing and length of smoking breaks. If smoking shelters are provided they are required by law to not be 'substantially enclosed'. Definitions, guidance and information on shelters are available on the internet (e.g. www.smokefreeengland.co.uk). Other useful sources are listed in Box 3.

In a total ban, patients and staff who smoke outside premises can create a public nuisance. Complaints can be reduced by providing smoking shelters and waste bins at boundaries of grounds. Safety issues for night staff may arise if they take smoking breaks in isolated areas or places with a high crime rate. With fewer staff on duty at night, it is beneficial to plan arrangements for breaks for night staff, in advance. 


\section{Box 2 Comparison of total and partial bans in mental health services}

The information below is partly based on a paper by Lawn \& Pols (2005), which reviews more than twice as many partial smoking bans as total bans. There are no studies comparing partial and complete bans in the same population.

Total ban - buildings and grounds

- Simpler to implement

- Smoke-free area is defined by boundaries of the grounds

- Lower levels of aggression and use of seclusion

- Fewer 'discharge against medical advice'

- Less use of 'as required' medication

- Less risk of passive smoking

- Patients have to quit smoking if they are unable to leave the buildings

- More open to legal challenge

- Smokers who smoke outside the grounds may cause a nuisance to the general public

Partial ban - buildings only

- Similar to restrictions enshrined in legislation; permitting smoking in outside areas

- Easier to enforce in settings with ready access to grounds

- Allows patient choice whether to continue to smoke

- Problems if access to safe outdoor areas is restricted

- Increased costs of providing staff to supervise outside smoking breaks

- Increased risks for staff isolated with patients in grounds

- Staff may be exposed to tobacco smoke outside

- Risk increased if patients retain tobacco and sources of ignition

- Increased risk of fire from covert smoking indoors

- In forensic settings, increased security risks from patients mingling in groups outside

- Patients detained under mental health legislation may not be able to gain access to grounds

- Patients are less likely to quit smoking, with continuing health risks

\section{Box 3 Useful sources for smoking cessation support and information}

- Action on Smoking and Health (ASH UK): www.ash.org.uk

- Smoke Free England (its resource library contains documents giving guidance on legislation): www. smokefreeengland.co.uk.

- International Network on Smoking and Mental Health: www.publichealthzone.net/network/ index.html

- Mental Health Foundation:www.mentalhealth.org.uk

- NHS Stop Smoking Campaign: www.givingupsmoking.co.uk

- National Institute for Mental Health in England: www.nimhe.csip.org.uk

Other websites that have useful documents

- Checklist for a Smokefree Mental Health Trust: www.southwest.csip.org.uk/our-work/mentalhealth/mental-health promotion/public-health/public-health-portfolio-downloads

- Guidance for smoke-free services for Ireland: www.otc.ie

- Guidance for smoke-free services for England: www.smokefreeengland.co.uk

- Guidance for smoke-free services in Scotland: www.clearingtheairscotland.com

- Regulations for smoking bans in Wales: www.smokingbanwales.co.uk

- Smoke Free London's 'Smoke Free Policy and Interventions in Mental Health Settings: A Toolkit': www.smokefreelondon.com/article.php?id=107

Publications

- Jochelson \& Majrowski (2006) Clearing the Air: Debating Smoke-Free Policies in Psychiatric Units

- Mental Health Foundation (2007) Taking a Deep Breath: The Mental Health Implications of Anti-smoking Legislation 


\section{Provision of tobacco}

The smoke-free policy should state that staff must not provide tobacco or purchase it for patients. Staff must not use tobacco to reward or punish patients and they should avoid promoting or advocating smoking.

\section{Smoking cessation}

Staff should ascertain the smoking status of all patients, record this in each patient's clinical records and encourage patients to reduce smoking or to quit. In the community, staff should ask patients and their carers to refrain from smoking in their presence. Patients in the community should be informed that in-patient settings are smoke-free. The policy should state the support available to help staff quit.

\section{Exemptions to the policy}

In England, the Healthcare Commission (2006) set exemption criteria for use on a case-by-case basis for 'long-stay mental health patients in an acute psychiatric state or terminally ill patients'. An 'acute psychiatric state' is not defined and is open to interpretation (Box 4). Clinical staff will need training in how to manage patients who challenge the policy. Staff should have consistent support from management and senior clinical staff, to avoid the granting of significant numbers of exemptions. It is advisable to monitor the number of exemptions and to address local concerns. In all cases, for exemptions to a smoke-free policy to be legal, they may permit smoking only outside in non-enclosed areas.

\section{Breaches}

In England, the responsibility for enforcing smokefree legislation lies with local authorities, who have powers to impose substantial fines or penalties on any organisation that allows smoking within its indoor premises. Similarly in Scotland and Ireland, fines can be imposed if smoke-free legislation is contravened.

The smoke-free policy must state what action should be taken when anyone smokes, and to whom to report the incident. Different procedures will be needed to manage breaches by staff, patients and visitors, taking care to balance the rights of each group.

Two strategies are effective in reducing the number of exemptions and breaches to a smoke-free policy: first, a clear communication strategy and second, widespread staff training in smoking cessation support.

Local services should develop their own procedures for breaches. Existing human resources or clinical policies that address related issues (such as
Box 4 Things to consider regarding exemptions from a smoke-free policy

- No blanket exemptions for categories of patients

- Long-stay psychiatric patients in an 'acute psychiatric state'

- Exemption on a case-by-case basis

- Who can make the decision to grant exemptions

- Where the grounds for exemption and the decision should be recorded

- How frequently the exemption should be reviewed

- What smoking cessation support should be offered to patients

- Arrangements for exempted patients to smoke

- Nursing care plans for exempted patients

(Healthcare Commission, 2006)

the consumption of alcohol or illicit drugs) can be adapted for the policy. Management can be lenient initially, as early breaches are likely to be unintentional, reserving disciplinary action for later.

\section{Maintaining a smoke-free environment}

Policies should state that there should be named members of staff responsible for maintaining the smoke-free environment and outline responsibilities of service user representatives. Sufficient staff must be trained as smoking cessation advisors at the appropriate level, and staff support systems should be introduced (Bloor et al, 2006).

\section{Policy implementation}

When the policy has been ratified, a date should be set for implementation, allowing at least 3 or 4 months to make the necessary preparations. Longer lead times can result in loss of motivation and momentum. Prominent leadership from management is essential at all stages.

\section{Management team}

Trust or central management

Introducing a smoking ban in mental health settings requires 'patience, planning and a bit of nerve' (Beemer, 1993). An executive lead should be given responsibility for the project, with assistance from a team that includes a project manager, an administrator, communications staff and clinicians with expertise in smoking cessation. 
First, the executive lead should convene a meeting with managers and senior clinicians from local services, to discuss how to implement the policy. Initial misconceptions about the policy can easily turn into fears about how local services will cope with the challenges. It is helpful if this meeting includes staff who have expertise in smoke-free policy implementation and smoking cessation services.

Thereon, the team should meet every 1 or 2 weeks, to manage the implementation. Additional financial and human resources will be needed for staff training, material/structural changes (e.g. no-smoking signs) and for provision of resources. Training and resources should be shared to avoid duplication of effort.

\section{Local management}

In local services, staff responsible for smoke-free policy implementation should communicate regularly with the main management team. In small units, one or two staff can lead the implementation of the policy. In larger services, a staff team will be needed comprising general managers, facilities / hotel services staff, patient advocates, occupational therapists, medical and nursing staff, and union representatives, in addition to occupational health nurses and staff from communications, pharmacy and security (in forensic settings).

\section{Working groups}

Working groups can undertake specific tasks such as surveys of patients and staff, developing resources to support smoking cessation, arranging staff training and supporting clinical teams. Working groups should liaise with others such as patients' representatives, clinical teams, nursing staff, physical health services, pharmacy, audit and management teams.

\section{Surveys of patients and staff}

Before undertaking surveys, it is essential to have managerial support, and to obtain a database of contact details for questionnaire recipients, together with administrative assistance for printing, filling envelopes and postage. It is best if completed questionnaires can be read electronically, to facilitate data processing and analysis.

Surveys give patients and staff an opportunity to express views anonymously and much the same survey can be carried out after the policy has been introduced, to gauge its impact (see 'Evaluating and revising the policy' below). Surveys can incorporate questions on attitudes of both smokers and nonsmokers towards the policy, the level of addiction of smokers and their need for support, and space for comments. Findings should be shared with patients, staff and management teams.

\section{Communication}

In the UK and Ireland, smoke-free regulations set legal requirements covering communication, for example the size of no-smoking signs, with which it is advisable to comply.

Smoke-free communication strategies should aim to reach patients and staff, in both in-patient and community settings. A senior manager should write to each in-patient about the policy, with the date and time of policy implementation, so that patients can choose whether to quit smoking in advance or at the last minute.

At entrances, a 'count-down' sign can be displayed showing the number of days remaining until the smoke-free date. This may be unpopular but is helpful in raising awareness of the immediacy of the ban.

Clinical staff must be kept informed about developments and provision of resources. Training should be provided to enable staff to manage the transition to a smoke-free environment. In Nottinghamshire Healthcare NHS Trust, we used the communication strategy outlined in Box 5. A 'smoke-free' icon on our intranet homepage led to smoke-free resources and information such as a 'Question and Answer' document that provided reasons for the policy, answered frequently asked questions and helped staff to facilitate discussions with smokers. Later, the intranet gave access to all smoke-free resources produced throughout the trust. We also had an email link to managers so that comments and questions could be addressed promptly.

\section{Smoking cessation support}

Pharmacological support

Pharmacy services should purchase sufficient stocks of medication to support smoking cessation for inpatients. Pharmacy staff can help clinical teams by providing information on the medication available to help patients to quit smoking, as detailed by Luty (2002). Awareness should be raised about possible changes in mental state after smoking cessation, for example a low mood or anxiety.

\section{Nicotine replacement therapy}

Nicotine replacement therapy (NRT) involving nasal sprays, gum or lozenges provides the fastest rise in nicotine blood levels and tends to suit those most heavily addicted to tobacco. Nasal and oral nicotine medications such as lozenges and gum should initially 


\section{Box 5 Communication strategy}

Routes and systems

- Letters: to each patient, giving the date and plans to become a smoke-free organisation

- Count-down sign: at entrances, giving days remaining to the quit date

- Existing pathways: notice boards, team briefings, newsletters, signs, emails

- Intranet: smoke-free logo linking to resources for smoke-free implementation

- Emails: to staff on progress and available resources

- Teleconference and conference telephone calls between central and local teams

- Signs: must comply with government regulations

Face to face

- Informal conversations: colleagues, clinical teams, patients and carers

- Forums: patients and staff in community and in-patient settings, carers' meetings, postgraduate meetings: present research and facilitate discussion

- Staff meetings: regular and extra meetings to discuss the implementation

- Ward visits: support for staff and guide to 'cold spots' with little or no action taken

- Ambassadors: support for local services from senior nurses

Information pack for wards and medical staff

- Copy of intranet home page, showing the smoke-free icon for access to information

- Support manual for staff on smoking cessation support

- Template for nursing care plans and quit records

- Question and answer document

- Posters for ward notice boards for patients

- Pharmacy information pack

External communication

- Press release for local newspapers and radio stations

- Information for local voluntary sector organisations

- Information for new patients and carers

- Advertisements for new employees should state that the organisation is smoke-free

- Information on the policy and procedures should be available to all staff

- Information for staff living on-site or in hospital property in shared accommodation

- Information for contractors and visitors

(Based on the strategy used by Nottinghamshire Healthcare NHS Trust in 2007 to implement their smoke-free policy in grounds and buildings) be taken frequently (e.g. hourly), so for in-patient settings it is best to use a medication administration chart designed for frequent administration. Nicotine patches, although providing a less rapid nicotine dose than nasal sprays or gum, are simpler to administer and use.

Whenever possible, patients should choose their preferred nicotine product, although choice may be influenced by factors such as the level of nicotine dependency or security issues, for example NRT gum is prohibited in high secure settings. Patients and staff should be aware that, although NRT can reduce cravings and make quitting easier, it does not eliminate the desire to smoke (McNally, 2006).

Nicotine replacement products are traditionally offered only to those intending to quit smoking completely. However, with responsible prescribing, they can also be used to ease the withdrawal symptoms of those not wishing to quit but unable to smoke for long periods. A report from the Royal College of Physicians (2007) notes the great potential for harm reduction of making nicotine supplements more available to smokers reducing (but not quitting) smoking and, more generally, highlights the irony of the disproportionate regulations applied to the safer nicotine replacement products, in comparison with the regulations applied to dangerous tobacco products.

Patients may make more than one attempt to quit and should be offered NRT or other medication to support each attempt. Patients should be encouraged to tell their clinical team when beginning smoking cessation, so that monitoring and support can be provided. When in-patients defer smoking cessation until the deadline, it is best to prescribe NRT in advance, to avoid overwhelming the on-call medical services.

\section{Bupropion}

Originally developed as an antidepressant, bupropion is an effective aid to smoking cessation, with efficacy comparable with that of NRT (Hughes et al, 2000). However, bupropion is rarely used in UK mental health settings because it has the potential to lower the seizure threshold. Use of bupropion is contraindicated with the concomitant use of some types of antipsychotic and antidepressant medications (Breckenridge, 2001).

\section{Varenicline}

Varenicline was approved for use as an aid to smoking cessation in 2006. Its mode of action is to bind to nicotinic receptors ( $\alpha_{4} \beta_{2}$ subtype), which are involved in dopamine production. Varenicline binds preferentially to nicotinic receptors, reducing the amount of additional dopamine released when a person smokes, thus reducing to a certain extent the 
reward from smoking and cravings in abstinence. Varenicline has been shown to be an effective adjunct to smoking cessation in the short term and at 1-year follow-up (Gonzales et al, 2006; Jorenby et al, 2006). Since trials of varenicline have so far excluded participants with a mental illness, its safety and efficacy in psychiatric patients remains unclear.

\section{Psychotropic medication and smoking cessation}

Pharmacy staff and clinicians should raise awareness about the possible deleterious effects of smoking cessation on psychotropic medication. Hydrocarbon by-products of smoking induce liver enzyme production of cytochrome P450 enzymes 1A2 (CYP1A2) (Bazire, 2005). These enzymes are involved in the metabolism of some forms of psychotropic medication and consequently smokers often require higher doses. Smoking cessation leads to a fall in CYP1A2 enzymes within days of quitting, leading to an increase in serum levels of some medication. For example, abrupt cessation of smoking cannabis and tobacco has led to toxicity with olanzapine and clozapine (Zullino et al, 2002).

In addition to clozapine and olanzapine, other medications that are affected include diazepam, haloperidol, mirtazapine, theophylline and propranolol (Health Development Agency, 2004). Serum levels of clozapine can rise 1.5 times in 2-4 weeks after tobacco smoking cessation (de Leon, 2004) and each individual should be closely monitored. If possible, baseline serum levels of clozapine should be obtained before quit attempts. During smoking cessation, patients and staff should check for signs of toxicity such as drowsiness or myoclonic spasms. The dose of clozapine should be reduced and serum levels monitored more frequently. Conversely, if a patient starts smoking, the dose of medication may have to be increased.

\section{Communication}

On admission and discharge from hospital, the patient's general practitioner and the community team should be informed about the individual's smoking status and possible effects of smoking on medication.

\section{Psychological support for patients and staff}

During the intended move to a smoke-free policy, it is best to avoid presenting the smoke-free initiative as something 'handed down' from a senior level or as a 'tick box exercise' imposed by government.
Within the smoke-free debate, the themes of 'control' and 'rights' are largely unavoidable. Discussions are likely to focus on the rights of non-smokers, even if they are in a minority, to live or work in a safe and smoke-free environment, and the counter-arguments about the rights of smokers to light up a cigarette.

Awareness of the health risks associated with smoking can be raised by the distribution and display of educational literature and in-house publications. An outside expert can be invited to speak to patients and staff. Ventilation and air filtration systems have been shown to be ineffective in removing tobacco smoke from buildings (Leavell et al, 2006). It may therefore be worth conducting air-quality tests in wards to demonstrate the level of exposure of staff and patients to passive smoking (Alley \& McNally, 2006).

Exposure to secondhand smoke is best framed within the context of a wider initiative of health promotion. This encourages patients and staff to take ownership of the policy and tends to encourage staff to learn new skills and approaches to mental healthcare (Lawn \& Pols, 2005).

\section{Training staff in smoking cessation support}

In the UK, the NHS, commercial and charity organisations offer training at three levels. Level 1 training includes brief interventions and referral, whereas levels 2 and 3 focus on delivery of 'intensive' quit support over several sessions. Level 2 focuses on delivery on a one-to-one basis and level 3 relates to groups. National Health Service Stop Smoking Services report that with these methods, over $50 \%$ of clients who set a quit date were abstinent from smoking 4 weeks after the end of treatment (Health and Social Care Information Centre, 2005). Spontaneous attempts to quit have been found to be more successful at 6 months than planned attempts to quit later (West \& Sohal, 2006).

One of the aims of staff training in mental health settings should be to change the 'hearts and minds' of clinical staff by encouraging a more positive attitude towards providing smoking cessation support. This can be achieved with level 1 training, which the majority of staff will receive. This is often the only training that the less enthusiastic staff will attend, making these sessions important vehicles for culture change.

Health professionals in the UK who are not smoking cessation specialists usually train to level 2 (one-to-one support). However, most mental health professionals have the skills and necessary experience for therapeutic group work. By extending training to level 3, a more flexible and cost-effective support service can be delivered. The aim should 
be for the majority of staff to be trained in brief interventions (level 1), and selected staff from each clinical team to be trained in intensive quit support (levels 2 and 3).

Owing to the relatively rapid turnover of staff in mental health services, a training strategy that stops at delivering smoking cessation training at level 3 will be unlikely to achieve long-term provision of a smoking cessation support service. Periodically, an external training team should be employed to deliver a series of 'train the trainers' courses. This will also enable trainers to function as 'smoking cessation leads', capable of providing supervision and also coordination of quit support as well as liaison with local NHS Stop Smoking Services.

\section{Introduction of the policy}

Smoke-free policies can be introduced quietly by using the operational strategy in Box 6. A low-key approach is suitable in long-stay psychiatric units where the 'quit date' has been known for months and sufficient support has been provided to both patients and staff.

\section{Launch events}

An alternative strategy is to hold a launch event to raise awareness among staff and patients that the smoke-free policy has taken effect. Launch events provide opportunities to reinforce the rationale behind the smoke-free initiative and promote 'quit smoking' support, which tends to reduce the number of unintentional policy breaches and reduce the burden on staff responsible for maintaining the smoke-free environment.

At an organisational level, launch events should be publicised widely and offer refreshments. A member of the management team should talk about the aims of the new policy and answer questions, no matter how challenging. Other speakers could include a staff member from smoking cessation services, and one or two people who have successfully quit, as an inspiration to others. Ward or out-patient clinic events are an important supplement to the organisation's launch, and should focus on how the smoke-free policy will run in that particular setting.

\section{Staff providing direct patient care}

In the days immediately following policy implementation, breaches to the policy are inevitable. Mental health services will benefit from temporarily increasing staffing levels of nurses in the early days, especially on acute admission wards and in forensic settings. Medical staff can support nursing staff by prescribing NRT products promptly for newly admitted patients who are smokers.

\section{Box 6 Operational strategy}

In-patient services

- Consider a Saturday or Sunday as the implementation day because it will be quieter

- Ensure the full complement of nursing staff during the initial smoke-free period

- All smokers should have access to nicotine replacement therapy or other medication

- Provide extra diversionary activities for patients, e.g. carpet bowls, darts, board games

- Remove tobacco and ignition sources according to local procedures

- Manage those who refuse to hand in the above according to local procedures

- Provide leaflets on the policy to new admissions

- Arrange additional activities for the forthcoming weeks

Procedures

- Decide how to deal with contraband items in the unit

- Arrange management of tobacco and ignition sources for those exempted from the policy

Facilities

- No-smoking signs at all entrances, compliant with government regulations

- Lockers for smoking materials should be provided for staff and visitors at entrances

- Offer healthy options of fruit, drinks and snacks on the wards

- Decommission smoking rooms; remove ashtrays and ignition sources

- Decide whether shelters for patients and staff to smoke (within or near the boundary of the grounds) are needed

\section{Evaluating and revising the policy}

Lessons will be learned from the initial few months of maintaining a smoke-free mental health service. The implementation team should reconvene for an evaluation and revision exercise (Box 7).

Data collection for the evaluation should be planned in advance. The survey instrument administered before the implementation of the smoke-free policy can be adapted and used again around 6 months after implementation. The aim of data collection should be to collect a range of opinion and suggestions from both staff and service users.

Managers and clinicians should not be afraid to revise a smoke-free policy to incorporate 
Box 7 Monitoring smoke-free implementation

For patients

The number of:

- adverse incidents related to the smoke-free policy

- breaches to the policy

- exemptions to the policy

- patients who have quit smoking

- patients discharging themselves from hospital against medical advice

Visitors and carers

The number of:

- breaches to the policy

- untoward incidents involving the smokefree policy

For staff

The number of:

- breaches to the policy

- disciplinary proceedings instituted about the smoke-free policy

- staff trained to support patients with smoking cessation

Survey patients and staff

Before and after policy implementation, ask about:

- attitudes towards the smoke-free policy

- facilities for smoking, if provided

- smoking cessation support services

- medication to support smoking cessation

For management

Six months after implementation:

- Results of the post-ban survey

- Lessons learned during the implementation

- Number of staff trained in smoking cessation support

- Report to the organisation's board

- Assess the financial cost of policy implementation and continuing costs

- Review the smoke-free policy

suggestions. Although some research has been done in this area, this is still a new field of work. So organisations implementing smoke-free initiatives should recognise their role as pioneers and improve their smoke-free policies and procedures as their experience grows.

\section{Conclusions}

Those who quit smoking or reduce the amount they smoke will gain lasting benefits to their physical health. The environment will improve for nonsmokers, who will no longer be affected by the impact of passive smoking.

Mental health services in England have a short time to start to implement a range of strategies relating to smoke-free policies and smoking cessation support, before the deadline of 1 July 2008. Ironically, if anxieties about implementing a smoke-free policy lead to delays in planning and preparation, then it is highly likely that these anxieties will become a selffulfilling prophecy, particularly if a poorly conceived policy is delivered, without adequate support to staff or patients.

Early and effective action taken towards implementing a smoke-free policy is the best way to ensure that the benefits to staff and patients of a smoke-free environment are achieved while minimising the risks of negative consequences. Management teams and clinicians should work together to support patients and staff in these important changes.

\section{Declaration of interest}

None.

\section{References and related articles}

Alley, K. \& McNally, L. (2006) Communicating a Smoke Free Policy. Smokefree London (http://www.smokefreelondon.com/ xstandard $/ 13 \% 20 \% 20$ Fact $\% 20$ Sheet $\% 20-\% 20$ Communicating $\% 20 a \% 20$ Smoke-Free\%20Policy.pdf).

Bazire, S. (2005) Psychotropic Drug Directory. Fivepin.

Beemer, B. R. (1993) Hospital psychiatric units. Non-smoking policies. Journal of Psychosocial Nursing and Mental Health Services, 31, 12-14.

Bloor, R. N., Meeson, L. \& Crome, I. B. (2006) The effects of a non-smoking policy on nursing staff smoking behaviour and attitudes in a psychiatric hospital. Journal of Psychiatric and Mental Health Nursing, 13, 188-196.

Breckenridge, A. (2001) Zyban - Modified Dosage and Safety Precautions. Committee on Safety of Medicines.

*Campion, J., McNeill, A. \& Checinski, K. (2006) Exempting mental health units from smoke-free laws. BMJ, 333, 407-408.

*el-Guebaly, N., Cathcart, J., Currie, S., et al (2002) Public health and therapeutic aspects of smoking bans in mental health and addiction settings. Psychiatric Services, 53, 1617-1622.

Etter, M. \& Etter, J. F. (2007) Acceptability and impact of a partial smoking ban in a psychiatric hospital. Preventive Medicine, 44, 64-69.

de Leon, J. (2004) Atypical antipsychotic dosing: the effect of smoking and caffeine. Psychiatric Services, 55, 491-493.

Gonzales, D., Rennard, S. I., Nides, M., et al (2006) Varenicline, an ${ }_{42}$ nicotinic acetylcholine receptor partial agonist, vs sustainedrelease bupropion and placebo for smoking cessation. A randomized controlled trial. JAMA, 296, 47-55.

Griffiths, J. (2005) Smokefree Scotland: Guidance on Smoking Policies for the NHS, Local Authorities and Care Service Providers. Scottish Executive (http:/ / www.scotland.gov.uk/ Publications/2005/12/21153341/33417).

Harris, G. T., Parle, D. \& Gagne, J. (2007) Effects of a tobacco ban on long-term psychiatric patients. Journal of Behavioral Health Services and Research, 34, 43-55.

Health and Social Care Information Centre (2006) Statistics on NHS Stop Smoking Services in England, April 2005 to March 2006. The Information Centre (http://www.ic.nhs.uk/ webfiles/publications/sss0506/SSS $\% 20$ anual $\% 20$ bulletin $\% 20$ 2005-06\%20full\%20reportv2.pdf). 
Healthcare Commission (2006) Key Performance Indicators 2005/6: Smoke-Free NHS, Recording of Smoking Status and Reducing Smoking. Healthcare Commission (http://ratings2006. healthcarecommission.org.uk/Indicators 2006Nat/Trust/ Indicator/indicatorDescriptionShort.asp?indicatorId=5235).

Health Development Agency (2004) Smoking and Patients with Mental Health Problems. Health Development Agency.

Hughes, J. R., Stead, L. F. \& Lancaster, T. (2000) Anxiolytics and antidepressants for smoking cessation. Cochrane Library, issue 3. Update Software.

Irish Health Promotion Network (2006) Best Practice Guidelines to Support Compliance with National Policy in Relation to Tobacco Management in the Mental Health Setting. Irish HP Network.

Jochelson, K. (2006) Smoke-free legislation and mental health units: the challenges ahead. British Journal of Psychiatry, 189, 479-480.

Jochelson, K. \& Majrowski, B. (2006) Clearing the Air: Debating Smoke-Free Policies in Psychiatric Units. King's Fund.

Jorenby, D. E., Hays, J. T., Rigotti, N. A., et al (2006) Efficacy of varenicline, an nicotinic acetylcholine receptor partial agonist, vs placebo or sustained-release bupropion for smoking cessation. A randomized controlled trial. JAMA, 296, 56-63.

*Lawn, S. \& Pols, R. (2005) Smoking bans in psychiatric in-patient settings? A review of the research. Australian and New Zealand Journal of Psychiatry, 39, 866-885.

Leavell, N. R., Muggli, M. E., Hurt, R. D., et al (2006) Blowing smoke: British American Tobacco's air filtration scheme. BMJ, $332,227-229$.

Luty, J. (2002) Nicotine addiction and smoking cessation treatments. Advances in Psychiatric Treatment, 8, 42-48.

McNally, L. (2006) Smoking and Mental Health: Helping Smokers to Quit in Mental Health Settings. Gasp.

McNally, L., Oyefeso, A., Annan, J., et al (2006) A survey of staff attitudes to smoking-related policy and intervention in psychiatric and general health care settings. Journal of Public Health (Oxford), 28, 192-196. Epub June 29.

Meltzer, H., Gill, B., Petticrew, M., et al (1996) Economic Activity and Social Functioning of Residents with Psychiatric Disorders (OPCS Surveys of Psychiatric Morbidity in Great Britain, Report 6). TSO (The Stationery Office).

Mulcahy, M., Evan, D. S., Hammond, S. K., et al (2005) Secondhand smoke exposure and risk following the Irish smoking ban: an assessment of salivary cotinine concentrations in hotel workers and air nicotine levels in bars. Tobacco Control, 14, 384-388.

Mental Health Foundation (2007) Taking a Deep Breath: The Mental Health Implications of Anti-smoking Legislation. Mental Health Foundation.

Office for NationalStatistics (2007) Statistics on Smoking, England 2007. ONS (http: / / www.ic.nhs.uk/statistics-and-data-collections / health-and-lifestyles / smoking / statistics-on-smokingengland-2007-[ns])

Resnick, M. P. \& Bosworth, E. E. (1989) A smoke-free psychiatric unit. Hospital and Community Psychiatry, 40, 525-527.

Royal College of Physicians (2007) Harm Reduction in Nicotine Addiction: Helping People Who Can't Quit. Royal College of Physicians of London.

Trinkoff, A. M. \& Storr, C. L. (1998) Substance use among nurses: differences between specialties. American Journal of Public Health, 88, 581-585.

West, R. \& Sohal, T. (2006) "Catastrophic" pathways to smoking cessation: findings from national survey. BMI, 332, 458-460.

Zullino, D. F., Delesser, D., Eap, C. B., et al (2002) Tobacco and cannabis smoking cessation can lead to intoxication with clozapine or olanzapine. International Clinical Psychopharmacology, 17, 141-143.

*Articles of particular interest.

\section{MCQs}

1 Smoking among people with mental illnesses:

a is up to three times more prevalent than among the general population

b was less prevalent in 2007 than in 1997 c reduces the required dosage of olanzapine

d is associated with a lower rate of psychiatric hospital admissions

e is more prevalent among community-based patients than among in-patients.

2 Smoking bans on psychiatric wards:

a are illegal under Scottish legislation

$\mathrm{b}$ will inevitably lead to an increase in ward disruption

c will be a legal requirement under English law from July 2008

d have been found to lead to a decline in the mental health of in-patients

e must include hospital gardens and grounds.

3 Regarding pharmacological smoking cessation aids:

a nicotine replacement therapy eliminates the desire to smoke

b nicotine replacement therapy can reduce the metabolism of clozapine

c bupropion can lower the seizure threshold

d varenicline contains nicotine

e pharmacological support should only be used in a patient's first attempt to quit.

4 Regarding the effects of smoking on medication metabolism:

a metabolism is affected only by heavy smoking

b nicotine replacement therapy increases the metabolism of clozapine

c blood serum levels of clozapine are likely to decrease on cessation of smoking

d patients taking clozapine should not stop smoking

e tobacco smoking induces cytochrome P450 enzyme production in the liver.

5 Regarding smoking cessation support:

a NHS Stop Services report a 4-week quit success rate of over $85 \%$

b level 1 support is offered only to those with high nicotine dependency

c smoking cessation support is inappropriate for patients under 18 years old

d successful quit rates in response to smoking cessation interventions within mental health settings are comparable to those observed among the general population

e smoking cessation support is less successful in forensic psychiatric settings.

\section{MCQ answers}

$\begin{array}{lllllll}1 & & 2 & & 3 & 4 & 5 \\ \text { a } & \text { T } & \text { a F } & \text { a F } & \text { a F } & \text { a F } \\ \text { b F } & \text { b F } & \text { b F } & \text { b F } & \text { b F } \\ \text { c F } & \text { c T } & \text { c T } & \text { c F } & \text { c F } \\ \text { d F } & \text { d F } & \text { d F } & \text { d F } & \text { d T } \\ \text { e F } & \text { e F } & \text { e F } & \text { e T } & \text { e F }\end{array}$

